\title{
Untersuchungen zur kombinierten Wirkung der Stressoren Lärm und Hitze auf Leistung, Stimmung und Sozialverhalten des Menschen
}

Manuela Lösch

\section{Zusammenfassung}

Für Deutschland werden in den nächsten Jahrzehnten ein starker Anstieg der Verkehrslärmbelastung und die Zunahme von Hitzeperioden prognostiziert. Lärm und Hitze beeinträchtigen je für sich nachweislich kognitive Leistungen und beeinflussen das Sozialverhalten, indem sie aggressive Reaktionen beschleunigen, ärgerliche Stimmungen verstärken und prosoziales Verhalten hemmen. Über die kombinierten Effekte von Lärm und Hitze auf kognitive Leistungen und Sozialverhalten liegen bis jetzt nur wenige und zudem widersprüchliche Erkenntnisse vor.

Ziel der vorliegenden Studie war es, die kombinierte Wirkung von Straßenverkehrslärm und Hitze auf kognitive Leistung, emotionales Erleben und Sozialverhalten zu untersuchen. Die kognitive Leistungsfähigkeit, Stimmung und Sozialverhalten werden mit Hilfe verschiedener kognitiver Aufgaben und Fragebögen erfasst.

In einer laborexperimentellen Untersuchung wurde die kombinierte Wirkung von Temperatur (in den Abstufungen 22 und $29^{\circ} \mathrm{C}$ ) und Straßenverkehrslärm (in den Abstufungen 37 und $70 \mathrm{~dB}(\mathrm{~A})$ ) analysiert. Es zeigte sich, dass Hitze die Hilfsbereitschaft der Teilnehmer reduziert. Hinsichtlich der kognitiven Leistung, der Bearbeitungszeiten im Leistungstest, der Stimmung sowie der subjektiven Bewertung konnten Interaktionen zwischen den Faktoren Temperatur und Straßenverkehrslärm nachgewiesen werden. 


\section{Theoretischer Hintergrund}

\subsection{Zukünftige Straßenverkehrslärm- und Hitzebelastung in Deutschland}

Eine aktuelle Umfrage im Auftrag des Bundesministeriums für Umwelt, Naturschutz und Reaktorsicherheit und des Umweltbundesamtes (UBA) ergab, dass sich gegenwärtig $54 \%$ der Befragten in ihrem Wohnumfeld durch Straßenverkehr gestört oder belästigt fühlen (Rückert-John, Bormann \& John, 2013). Straßenverkehr ist in Deutschland die dominierende Geräuschquelle. Fast 1,8 Millionen Menschen sind tagsüber mit Schallpegeln von über 65dB(A) belastet (UBA, 2010). Bis 2025 wird voraussichtlich im motorisierten Individualverkehr die Verkehrsleistung um ca. $16 \%$ und im Güterverkehr um $70 \%$ ansteigen (Intraplan Consult GmbH und BVU Beratergruppe Verkehr + Umwelt GmbH, 2007 im Auftrag des Bundesministeriums für Verkehr, Bau und Stadtentwicklung).

Auch mit einer Zunahme der Hitzebelastungen ist zu rechnen. Tinz, Freydank \& Hupfer (2008) sprechen von einer Hitzewelle in Deutschland, wenn an mindestens fünf aufeinanderfolgenden Tagen Temperaturen über $30^{\circ} \mathrm{C}$ erreicht werden. Aktuell lassen sich durch Auswirkungen des Klimawandels bereits auf fünf Prozent aller Landflächen monatliche Hitzeextreme im Sommer beobachten. Bis 2020 wird sich diese Zahl voraussichtlich verdoppeln und bis 2040 vervierfachen (Coumou \& Robinson, 2013). Oft führen Hitzeextreme zu Ernteverlusten, Waldbränden und Todesfällen in den aufgeheizten Städten. Im Hitzesommer 2003 waren in Deutschland rund 7000 hitzebedingte Todesopfer zu verzeichnen (Zebisch et al., 2005). Gemäß einer Studie im Auftrag des WWF Deutschland (Hübler \& Klepper, 2007) werden bis zum Ende dieses Jahrhunderts ca. 17000 durch Hitzeextreme bedingte Todesopfer pro Jahr prognostiziert.

\subsection{Lärmwirkungen}

Lärm ist unerwünschter Schall, der als lästig erlebt wird und Wohlbefinden, Leistung und Gesundheit sowie das soziale Zusammenleben der Menschen beeinträchtigen kann (Hellbrück, Guski \& Schick, 2010). Diese Definition impliziert, dass Lärm eine Reihe physiologischer, psychologischer, sozialer und sozio-ökonomischer Wirkungen auslöst (extraaurale Lärmwirkungen). Griefahn (2000) unterscheidet bei den extraauralen Lärmwirkungen zwischen primären, sekundären und tertiären Effekten. Primäre Lärmwirkungen ergeben sich unmittelbar auf einen akustischen Reiz und entstehen während der Lärmexposition. Dazu zählt eine durch Lärm ausgelöste 
Störung der Ruhe und/oder des Schlafes. Zudem kann Lärm Beeinträchtigungen der sprachlichen Kommunikation auslösen, indem laute tiefe Frequenzen (z. B. Abrollgeräusche auf der Straße) leise hohe Frequenzen (Konsonanten in der Sprache) maskieren. Des Weiteren kann Lärm in direkter Exposition eine Ablenkung der Aufmerksamkeit und Ausschüttung von Stresshormonen (Katecholaminen und Cortisol), die den Organismus in einen erhöhten Alarmzustand versetzen, bewirken.

Sekundäre Lärmwirkungen entstehen kumulativ nach bestimmter Einwirkzeit und innerhalb des zeitlichen Verlaufs der Lärmexposition. Dazu zählen u. a. das durch Lärm ausgelöste Gefühl der Belästigung (annoyance) und die Beeinträchtigung kognitiver Leistungen. Die bewusste Wahrnehmung, dass Gedanken und Handlungen durch Lärm gestört werden, bewirkt ein Gefühl von Verärgerung, Missmut und Unbehagen. Dabei wird Lärm vor allem dann als störend empfunden, wenn er in Konkurrenz zu gleichzeitig geplanten Handlungen steht. Belästigung zählt zu den häufigsten Lärmeffekten. Jedoch klärt der Schallpegel der Lärmquelle nur etwa $33 \%$ des Belästigungsurteils auf (Fields, 1993; Guski, 1999; Job, 1988). Lärm unterbricht kognitive Aufgaben, indem er die Aufmerksamkeit ablenkt. Dadurch erhöht sich die Anspannung (reaktive Anspannungssteigerung) mit der Folge, dass mehr kognitive Ressourcen benötigt werden, die für eine angemessene Bearbeitung der Aufgabe dann u. U. nicht zur Verfügung stehen (Hellbrück \& Liebl, 2008). Insbesondere bei Lärm, der starke temporal-spektrale Variabilität aufweist, kann Lärm sich auch direkt auf das Kurzzeitgedächtnis auswirken und die Fehlerrate in Kurzzeitgedächtnistests erhöhen. Hygge, Boman \& Enmarker (2003) untersuchten den Einfluss irrelevanter Sprache und Straßenverkehrsgeräuschen (je 62dB(A)) auf die Erinnerungs- und Abrufleistung gelesener Texte sowie auf die Aufmerksamkeit. Bei den Ergebnissen zeigte sich, dass sowohl Hintergrundsprechen als auch Straßenverkehrsgeräusche Textabruf sowie die Aufmerksamkeitsleistung beeinträchtigen. Es konnte jedoch kein Unterschied zwischen der Wirkung von Sprache und Straßenverkehrslärm nachgewiesen werden. Die Autoren argumentieren, dass die akustische Variation des Hintergrundschalls leistungsmindernd wirkt.

Unter tertiären Effekten versteht man chronische Lärmwirkungen, die durch jahrelange und kontinuierliche Lärmbelastung, beispielsweise bei Anwohnern stark befahrener Straßen, ausgelöst werden. Hierzu zählt auch die Ausbildung bestimmter Krankheitsbilder. Epidemiologische Untersuchungen deuten auf ein erhöhtes Risiko für Blutdruckerhöhungen und Herz-Kreislauferkrankungen sowie einen insgesamt erhöhten Medikamentenverbrauch in lärmbelasteten Gebieten hin (Bluhm, Nordling \& Berglind, 2001; Babisch, 2006).

Lärm wirkt sich auch auf das Sozialverhalten aus, indem er gewissermaßen als Katalysator ärgerliche Stimmungen verstärkt, aggressive Reaktionen beschleunigt und prosoziales Verhalten hemmt. Entsprechende Studien zeigten, dass Lärm di- 
rekt kein aggressives Verhalten auslöst, sondern nur dann, wenn die Teilnehmer bereits aus anderen Gründen verärgert waren. Dabei erhöht Lärm zusätzlich das allgemeine Erregungsniveau und kann „gewissermaßen den Tropfen darstellen, der das Fass zum Überlaufen bringt“" Wenn die Teilnehmer nicht in ärgerlicher Stimmung waren, so löste der Lärm nicht signifikant mehr aggressive Handlungen aus (Cohen \& Spacapan, 1984; Donnerstein \& Wilson, 1976; Geen \& O'Neal, 1969; Koneci, Libuser, Morten \& Ebbsen, 1975). Verschiedene sozialpsychologische Studien konnten nachweisen, dass sich Lärm auf die Hilfsbereitschaft auswirkt. Dabei zeigte sich, dass die Reduktion der Hilfsbereitschaft sowohl während der Lärmexposition (Mathews \& Canon, 1975) als auch danach (Sherrod \& Downs, 1974) auftritt. Ursache hierfür kann die Missstimmung sein, die durch Lärm ausgelöst wird. Fühlen sich Menschen durch Lärm gereizt bzw. verärgert und sind dadurch in schlechter Stimmung, sinkt die Wahrscheinlichkeit, dass sie ihren Mitmenschen helfen. In mehreren Untersuchungen zeigte sich, dass nach Beschallung durch Lärm prosoziales Verhalten erheblich reduziert wird (Korte \& Grant, 1980; Sherrod \& Downs, 1974). In jüngerer Zeit sind kaum mehr wissenschaftliche Untersuchungen zur Wirkung von Lärm auf das Sozialverhalten zu registrieren.

\subsection{Temperaturwirkungen}

Als homoiothermes (gleichwarmes) Lebewesen ist der Mensch in der Lage, die Kerntemperatur des Körpers auf etwa $37^{\circ} \mathrm{C}$ konstant zu halten. Die Thermoregulation sorgt mit Hilfe adaptiver Mechanismen dafür, dass Wärmeaufnahme und Wärmeabgabe im Gleichgewicht stehen und somit die Kerntemperatur des Körpers konstant bleibt. Der Prozess der Thermoregulation kann als Kontrollschleife betrachtet werden (Werner, 2005). Über periphere Thermorezeptoren der Haut laufen im vorderen Hypothalamus alle Temperaturinformationen aus der Haut, dem Inneren des Körpers und den Muskeln zusammen. Über metabolische Mechanismen (Schweißproduktion, periphere Vasodilatation, Konvektion) wird der Wärmehaushalt des Menschen im Gleichgewicht gehalten. Eine Überwärmung des Körpers führt zu Ohnmacht, Übelkeit, Erbrechen, Kopfschmerzen, Ruhelosigkeit und unter Umständen zu einem Hitzschlag. Bei normalem Gesundheitszustand treten jedoch bei anhaltend hoher Umgebungstemperatur Akklimatisierungsmechanismen ein.

Überblicksarbeiten zum Einfluss von Hitze auf kognitive Leistung (Hancock, Ross \& Szalma, 2007; Pilcher, Nadler \& Busch, 2002; Seppänen, Fisk \& Faulkner, 2004; Seppänen, Fisk \& Lei, 2006) weisen nach, dass Hitze bei verschiedenen Testverfahren zu generellen Leistungseinbußen führt. Gleichzeitig zeigen die Autoren, 
dass die Faktoren Intensität des Stressors Hitze, Aufgabentyp und Expositionsdauer die Temperaturwirkung mit beeinflussen können. Hinsichtlich der Intensität des Stressors zeigen die Meta-Analysen, dass ab einer Temperatur von ca. $25^{\circ} \mathrm{C}$ die Leistungsminderung mit steigender Temperatur zunimmt. Im Bereich zwischen $21^{\circ} \mathrm{C}$ und $25^{\circ} \mathrm{C}$ können keine Temperatureffekte nachgewiesen werden. Nach Hancock et al. (2007) steigt die Leistungsverschlechterung ab $29,4^{\circ} \mathrm{C}$ exponentiell an, da ab dieser Temperatur die Thermoregulation des Menschen gestört wird. Bezüglich des Einflussfaktors Aufgabentyp zeigte sich, dass es bei Verfahren zur Untersuchung von Wahrnehmung, Aufmerksamkeit und Psychomotorik unter erhöhter Raumtemperatur zu stärkeren Leistungsverschlechterungen kommt als bei Verfahren, welche mathematische Fähigkeiten, Reaktionszeiten, Schlussfolgern, Lernen und Gedächtnis untersuchen. Seppänen et al. (2004) berichten, dass ab $25^{\circ} \mathrm{C}$ die kognitive Leistung pro $1^{\circ} \mathrm{C}$ Erwärmung um $2 \%$ sinkt.

Eine unter dem Schlagwort Hitzehypothese bekannt gewordene These besagt, dass mit steigender Temperatur aggressives Verhalten zunimmt (Anderson, Anderson, Dorr, DeNeve \& Flanagan, 2000). Carlsmith \& Anderson (1979) haben festgestellt, dass in den 1960er und 1970er Jahren Unruhen und Krawalle in den USA wesentlich häufiger an heißen Tagen stattfanden als an kalten. Anderson et al. (2000) prognostizierten für die USA mit ihren ca. 270 Mio. Einwohnern, dass bei einer Zunahme der Durchschnittstemperatur um $1^{\circ} \mathrm{C}$ die Rate für Mord und Körperverletzung um 9 Fälle pro 100000 Menschen ansteigen wird. Bei einer Einwohnerzahl von 285 Millionen Menschen in den USA bedeutet dies, dass jährlich ca. 24.000 Gewaltdelikte mehr geschehen. Der Einfluss hoher Temperaturen auf Aggression kann mithilfe des allgemeinen Aggressionsmodells (Anderson et al., 2000) erklärt werden. Wird Hitze als unangenehm und belästigend empfunden, löst dies negative affektive Erregungen aus. Diese beeinflusst wiederum die kognitive Verarbeitung und Bewertung sozialer Stimuli und vergrößert so die Wahrscheinlichkeit aggressiven Verhaltens (Krahé, 2007). Allerdings sind die Zusammenhänge nicht so einfach, wie es vielleicht erscheinen mag.

Laborexperimentelle Untersuchungen zeigen, dass Hitze einerseits bei verärgerten Personen aggressives Verhalten reduziert, aber andererseits diese Verhaltensweisen bei nicht-verärgerten Personen steigert (Baron \& Bell, 1975; Baron \& Bell, 1976, Exp. 1). Baron \& Bell (1976) erklären diesen Zusammenhang mit Hilfe des „Negative Affect-Escape Models“. Demnach lösen sowohl die Hitze als auch die Verärgerung bei der Versuchsperson negative Emotionen aus. Diese Emotionen wirken als Mediator im Zusammenhang zwischen Hitze und Aggression. Man vermutet einen kurvilinearen Zusammenhang zwischen Hitze und Aggression im Sinne einer umgekehrten-U-Funktion. Hitze löst negative Emotionen aus. Diese lassen Aggressionen bis zu einem kritischen Punkt ansteigen. Werden jedoch die 
Emotionen stärker, so reduziert sich aggressives Verhalten wieder. In Hinblick auf den Zusammenhang zwischen Hitze und Aggression zeigen diese Erkenntnisse, dass aggressive Handlungen innerhalb eines kritischen Bereichs unbehaglich hoher Umgebungstemperatur leichter auftreten können. Andererseits führt die Kombination von extrem hoher Umgebungstemperatur und Verärgerung dazu, dass Aggressionen reduziert werden, da die Personen mehr darauf konzentriert sind, der Hitze zu entkommen. Demnach löst Hitze allein - ähnlich dem Lärm noch kein aggressives Verhalten aus.

Die sozialpsychologischen Studien, die zur Untersuchung des Einflusses von Hitze auf die Hilfsbereitschaft durchgeführt wurden, zeigten bis jetzt noch inkonsistente Ergebnisse. In einigen Untersuchungen konnte eine Reduktion der Hilfsbereitschaft bei hoher Umgebungstemperatur nachgewiesen werden (Page, 1978; Cunningham, 1979). In anderen Studien wurde dagegen kein Zusammenhang zwischen Hitze und verminderter Hilfsbereitschaft nachgewiesen (Schneider, Lesko \& Garret, 1980).

\subsection{Kombination von Lärm und Hitze}

Zahlreiche Studien untersuchten die kombinierten Wirkungen der Stressoren Lärm und Hitze auf die menschliche Leistung (Hancock \& Pierce, 1985; Horie, Sakurai, Noguchi \& Matsubara, 1985; Hygge \& Knez, 2001; Pellerin \& Candas, 2003, 2004), jedoch mit widersprüchlichen Ergebnissen.

Überblicksarbeiten (Grether, 1970; Hancock \& Pierce, 1985; Murray \& McCally, 1973) zeigen, dass Lärm und Hitze meist unabhängig voneinander wirken und es zu keinen Interaktionen zwischen den Stressoren kommt (Viteles \& Smith, 1946; Pepler, 1960; Bell, Provins \& Hiorns, 1964). Liegen jedoch Interaktionen vor, so treten sowohl additive als auch synergetische sowie antagonistische Effekte auf. Bei additiven Interaktionen ist die kombinierte Wirkung von Lärm und Hitze genauso groß wie die Summe der Einzeleffekte. Nach Broadbent (1971) ist bei synergistischen Effekten die kombinierte Wirkung zweier Umweltstressoren größer als die Summe der Effekte der einzelnen Stressoren. Renshaw (1971) zeigte synergistische Effekte zwischen Lärm und Hitze. Hier waren die Auswirkungen der Umgebungstemperatur auf die Leistungen der Teilnehmer abhängig vom Hintergrundschall.

Bei antagonistischen Effekten haben die Einzelstressoren jeweils eine entgegengesetzte Wirkung und somit heben sich bei einer Kombination der beiden Stressoren die Wirkungen der Einzelstressoren gegenseitig auf. Antagonistische Interaktionen zwischen Lärm und Hitze können mit Hilfe der Arousal Theorie erklärt werden. Demnach herrscht zwischen Erregung und Leistung ein kurvilinearer Zusammenhang im Sinne der umgekehrten-U Funktion. Ein mittleres 
Erregungsniveau ist dabei leistungsoptimierend, während eine zu hohe oder niedrige Aktivierung die Leistungsfähigkeit beeinträchtigt. Es wird angenommen, dass Lärm das Erregungsniveau steigert, wohingegen ab einem unkomfortablen Bereich $\left(\mathrm{ab} 27^{\circ} \mathrm{C}\right)$ das Erregungsniveau sinkt. Bei Kombination der beiden Stressoren entsteht somit ein mittleres Erregungsniveau, welches für optimale Leistung sorgt. Folglich kommt es gemäß der Arousal-Theorie bei Kombination von Lärm und Hitze zu Leistungsverbesserungen, welche die antagonistischen Effekte erklären. Hygge (1991) berichtet von antagonistischen Interaktionen zwischen Lärm (37 und $70 \mathrm{~dB}(\mathrm{~A}))$ und Temperatur $\left(20\right.$ und $\left.27,8^{\circ} \mathrm{C}\right)$ bei Problemlöseaufgaben. Hygge und Knez (2001) konnten zeigen, dass Hitze und Lärm von hoher Intensität (27 $\left.{ }^{\circ} \mathrm{C} / 58 \mathrm{~dB}(\mathrm{~A})\right)$ zu Leistungsverschlechterungen im Vergleich zur Kontrollbedingung $\left(21^{\circ} \mathrm{C} / 38 \mathrm{~dB}(\mathrm{~A})\right)$ führten. Dagegen führten Hitze und Lärm von niedriger Intensität $\left(27^{\circ} \mathrm{C} / 38 \mathrm{~dB}(\mathrm{~A})\right)$ zu Leistungsverbesserungen im Vergleich zur Kontrollbedingung.

Bei Witterseh, Wyon \& Clausen (2004) kam es zu einer antagonistischen Interaktion zwischen Bürolärm (Lärm aus Großraumbüro mit $55 \mathrm{~dB}(\mathrm{~A})$ ) und Temperatur $\left(22,26\right.$ und $\left.30^{\circ} \mathrm{C}\right)$. So fiel der Einfluss von Temperatur auf die Fehlerrate beim Additionstest in Anwesenheit von Bürolärm geringer aus als der Einfluss von Temperatur ohne gleichzeitigen Lärm.

Ziel einiger Studien war es, einen quantitativen Zusammenhang zwischen den Wirkungsweisen von Lärm und Hitze herzustellen. Clausen, Carrick, Fanger, Kim, Poulsen \& Rindel (1993) zeigten, dass eine Abweichung von der präferierten Temperatur um $1^{\circ} \mathrm{C}$ etwa einer Erhöhung der Lärmintensität um $4 \mathrm{~dB}(\mathrm{~A})$ entspricht. Nach Gunnarsen \& Santos (1998) verringert sich bei den Probanden das Gefühl der Belästigung bei einer Temperaturreduktion um $1^{\circ} \mathrm{C}$ genauso wie bei einer Reduktion der Schallintensität um $7 \mathrm{~dB}(\mathrm{~A})$.

Über die kombinierte Wirkung von Lärm und Hitze auf das Sozialverhalten liegen bisher nur sehr wenige Studien mit insgesamt widersprüchlichen Ergebnissen vor. Bell (1980) untersuchte die kombinierte Wirkung der Faktoren Temperatur $\left(21^{\circ} \mathrm{C}-23^{\circ} \mathrm{C} / 33^{\circ} \mathrm{C}-35^{\circ} \mathrm{C}\right)$ und Lärm $(55$ und $95 \mathrm{~dB}(\mathrm{~A}))$ auf das Sozialverhalten der Teilnehmer. Es wurde gezeigt, dass Lärm allein sich nicht auf das Sozialverhalten der Teilnehmer auswirkt. Wurden die Teilnehmer vor der Untersuchung vom Experimentator provoziert, so zeigten sie in der Hitzebedingung am häufigsten aggressives Verhalten. Bei Bell \& Doyle (1983) kommt es bei der Kombination von Lärm ( 55 und $95 \mathrm{~dB}(\mathrm{~A}))$ und Temperatur $\left(22,8\right.$ und $\left.35^{\circ} \mathrm{C}\right)$ weder zu Einzelwirkungen noch zu Interaktionen auf das Hilfsverhalten.

Wie oben berichtet, gibt es eine Vielzahl von Untersuchungen zu Lärm- und Hitzewirkungen. Diese sind jedoch zum Teil sehr alt und zeigen widersprüchliche Ergebnisse. Im Zuge des Klimawandels und der rasanten Verkehrsentwicklung werden diese Fragen aber wieder aktuell. 


\section{$2 \quad$ Ziel der Untersuchung}

Prognosen warnen, dass zukünftig das Aufkommen von Straßenverkehrslärm und Hitzeextremen steigen wird. Lärm und Hitze sind Stressoren, die Leistung, Emotionen, Sozialverhalten und Gesundheit in erheblichem Maße belasten können. Aus diesem Grund wird in den nächsten Jahrzehnten vermutlich sowohl die Lebensqualität und das Leistungsverhalten als auch die physische und psychische Gesundheit der Menschen in erheblichem Maße belastet werden. Gesundheit und Leistungsfähigkeit der Menschen sowie psychisches und soziales Wohlergehen sind schützenswerte Güter, denen im Sinne einer nachhaltigen gesellschaftlichen Entwicklung besondere Beachtung geschenkt werden muss. Zum gegenwärtigen Zeitpunkt bedarf es nach Lösungsansätzen, wie Menschen vor den in absehbarer Zukunft wahrscheinlich nicht mehr zu vermeidenden Belastungen, die mit Klimaveränderungen und Verkehrsentwicklungen verbunden sind, geschützt werden können. Dazu dienen die hier vorzustellenden Untersuchungen.

Ziel des Projekts ist es, die kombinierte Wirkung der Stressoren Lärm und Hitze auf die kognitive Leistung, die Stimmung und das Sozialverhalten zu untersuchen. Des Weiteren besteht mittels dieser Untersuchung die Chance dazu beizutragen, kommunalen Entscheidungsträgern, Behörden, Verkehrswegeplanern etc. mit Hilfe effektiver Kosten-Nutzen-Rechnungen Entscheidungshilfen beim Ergreifen von Maßnahmen zu geben, die Lärm- und Hitzestress vorbeugen.

\section{$3 \quad$ Allgemeine Methode}

\subsection{Abhängige Variablen}

Kognitive Leistung: Die Erfassung der kognitiven Leistungsfähigkeit erfolgte mit dem n-back Test. Beim n-back Test werden den Teilnehmern die Ziffern „1“ bis "9“ in einer fortlaufenden Reihe als Stimuli präsentiert. Innerhalb dieser Sequenz erscheinen Ziffern in roter Schrift. Die Teilnehmer sollen diesen Stimulus mit dem n-Stufen zuvor präsentierten Stimulus vergleichen. Die Lösung dieses Vergleichsprozesses (Übereinstimmung/fehlende Übereinstimmung) soll durch einen Tastendruck signalisiert werden. Je höher die n-Stufe, desto höher ist die Schwierigkeit des n-back Tests. In dieser Untersuchung werden die Stufen $n=4$ und n=5 gewählt. Pro Durchgang und n-Stufe werden je 34 bzw. 35 Stimuli präsentiert. Pro Zahlenreihe waren sechs Vergleichssituationen gefordert, die in zufälliger Reihenfolge auftraten. Postle, D'Esposito \& Corkin (2005) beschreiben, dass bei der 
Ausführung des n-back Tests jeder Stimulus, der dem Teilnehmer in fortlaufender Reihe gezeigt wird, enkodiert wird. Die gespeicherte Repräsentation des Stimulus wird unter Abschirmung von Interferenzen durch sequentiell präsentierte Reize aufrechterhalten. Zudem erfolgt eine Lenkung der Aufmerksamkeit zurück zur Repräsentation des Stimulus, sobald dies in der Aufgabe erforderlich wird. Des Weiteren folgen ein Vergleichsprozess zwischen der Repräsentation und dem aktuell präsentierten Reiz sowie die Steuerung der Handlung anhand des Resultates des Vergleichs. Zudem erfolgt eine aktive Aussortierung der Repräsentation und Aktualisierung der Positionen, der Items, die noch im Arbeitsgedächtnis gespeichert sind.

Altruistisches Verhalten: Die Erfassung des altruistischen Verhaltens erfolgt durch das Ultimatumspiel, einem Verfahren, das u. a. in der Sozialpsychologie und in den Wirtschaftswissenschaften zur Erforschung des Altruismus beziehungsweise Egoismus eingesetzt wird. In diesem Rollenspiel muss ein Akteur (Proposer) einen bestimmten Teil eines ihm zur Verfügung gestellten Gutes (z. B. Geld) einem anderen Akteur (Responder) anbieten. Lehnt dieser den ihm angebotenen Teil ab, so muss auch der Proposer auf seinen Teil verzichten. Beide gehen leer aus. Nimmt der Responder an, so erhält er das Angebot und der Proposer erhält den restlichen Teil.

Die Teilnehmer sind während der Testung in der Rolle des Responders und erhalten insgesamt 82 Angebote von verschiedenen fiktiven Proposern, welche durch ein Bild am Bildschirm präsentiert werden. Der Teilnehmer muss sich darauf innerhalb von vier Sekunden entscheiden, ob er das Angebot annimmt oder ablehnt. Die Entscheidung erfolgt durch Tastendruck am PC. Es werden folgende Angebote präsentiert: Kategorie 1: $50 €-41 €$ (20 Angebote); Kategorie 2: $40 €-$ $31 €(20$ Angebote); Kategorie 3: $30 €-21 €(20$ Angebote); Kategorie 4: $20 €-10$ $€$ (22 Angebote). Kategorie 1 werden als „faire Angebote“, die Kategorie $2-4$ als „unfaire Angebote“ festgelegt.

In ihrer Rolle als Responder haben die Teilnehmer die Möglichkeit, durch Annahme eines Angebotes nicht nur sich selbst, sondern auch dem Proposer Geld zukommen zu lassen. Der Responder hat jedoch auch die Möglichkeit durch Ablehnung eines Angebotes zu verhindern, dass dem Proposer ein Geldbetrag zukommt. Man kann also zum einen die Annahme unfairer Angebote als altruistisches Verhalten interpretieren, da der Teilnehmer nicht nur an sich selbst denkt, sondern auch sein Gegenüber berücksichtigt. Hingegen zeigt die Ablehnung fairer und unfairer Angebote ein egoistisches Verhalten. Der Teilnehmer möchte seinem Gegenüber nichts Positives zukommen lassen.

Zudem wird ein zusätzlicher Teil des Ultimatumspiels durchgeführt, bei dem die Teilnehmer in der Rolle des Proposers sind. Sie müssen fiktiven Respondern, welche Ihnen am PC präsentiert werden, Angebote machen, wie viel sie von 100 Euro abgeben. Das Angebot muss zwischen 10 und 50 Euro liegen. Die Eingabe 
des Angebots erfolgt über den PC. Wie in Teil 1 müssen 82 Angebote abgegeben werden. Die Probanden erhalten jeweils Rückmeldung, ob die fiktiven Proposer ihr Angebot annahmen oder nicht.

- Hilfsbereitschaft: Zur Erfassung der individuellen Hilfsbereitschaft der Teilnehmer wird eine Täuschung vorgenommen. Die Teilnehmer werden am Ende der Untersuchung mit Hilfe eines standardisierten Textes vom Versuchsleiter gefragt, ob sie zusätzlich freiwillig einen Leistungstest für die Untersuchung eines Kollegen bearbeiten möchten. Den Probanden wird nahe gelegt, dass ihre Teilnahme von großer Bedeutung für die Untersuchung des Kollegen wäre und auch sehr dringlich sei. Mit Hilfe dieser freiwilligen, fiktiven Untersuchung soll der Einfluss der Stressoren Straßenverkehrslärm und Hitze auf die Hilfsbereitschaft der Teilnehmer untersucht werden. Bei der Aufgabe handelt es sich um die Pauli-Kraeplinsche Arbeitsprobe (Arnold, 1975) (im Folgenden kurz Pauli-Test genannt). Die Teilnehmer müssen hierbei fortlaufend zwei einstellige Zahlen addieren und die Einerstelle des Ergebnisses notieren. Es herrscht keine Zeitvorgabe, die Teilnehmer dürfen die Bearbeitung der Aufgabe aber jeder Zeit früher beenden. Die Probanden erhalten kein Honorar für diese zusätzliche Aufgabe. Die Bereitschaft der Teilnehmer, freiwillig an dieser zusätzlichen Untersuchung teilzunehmen, kann somit als Maß für Hilfsbereitschaft verstanden werden.

- Erregung und Verärgerung: Zur Erfassung der Stimmungsdimensionen Erregung und Verärgerung wurde ein Fragebogen mit 40 Eigenschaftswörtern und Feststellungen entwickelt. Die Items entstammen unterschiedlichen, standardisierten Testverfahren (Perceived Arousal Scale, Anderson, Deuser \& DeNeve, 1995), Eigenschaftswortliste EWL (Janke \& Debus, 1978), State-Trait-Ärgerausdrucks-Inventar STAXI (Schwenkmezger, Hodapp \& Spielberger, 1992), State Hostility Scale (Anderson et al., 1995). Die Antworten der Teilnehmer werden auf einer 21-stufigen Antwortskala erfasst („überhaupt nicht“-,äußerst“).

- Kontrollvariablen: Zur Erfassung der Kontrollvariablen „Soziale Orientierung“, „Erregbarkeit" und „Aggression“ waren vor Untersuchungsbeginn die drei gleichnamigen Skalen des Freiburger Persönlichkeitsinventars FPI-R (Fahrenberg, Hampel \& Selg, 2001) zu bearbeiten. Zusätzlich wurde mithilfe des 'Noise Sensitivity Questionnaire' (NoiSeQ) (Schütte \& Mark, 2004) die jeweilige Lärmempfindlichkeit der Teilnehmer erfasst. Ebenso sollte die jeweilige Temperaturempfindlichkeit erfasst werden. Die dargebotenen Schalle und die Raumtemperatur wurden hinsichtlich ihrer wahrgenommenen Eigenschaften (Lautheit, gefühlte Temperatur, subjektiv empfundener Raumkomfort) sowie ihrer subjektiv empfundenen Störwirkung beurteilt. 


\subsection{Versuchsablauf}

Einige Tage vor der Testung erfolgt die Erfassung der allgemeinen Lärm- und Hitzeempfindlichkeit, des allgemeinen Gesundheitszustandes und der Persönlichkeitsskalen „Soziale Orientierung“, „Erregbarkeit“ und „Aggression“. In der Voruntersuchung wurden nur die Persönlichkeitsskalen „Soziale Orientierung“, „Erregbarkeit“ und „Aggression“ zu Anfang der Untersuchung erfasst.

Es soll zum einen garantiert werden, dass die die Kontrollvariablen „Lärmempfindlichkeit“, „Hitzeempfindlichkeit“, „Soziale Orientierung“, „Erregtheit“ und „Aggression“ in allen Teilnehmergruppen übereinstimmende Werte (im Rahmen der Messgenauigkeit) annehmen. Zusätzlich wurde der allgemeine Gesundheitszustand erfragt, damit keine kranken Personen an der Untersuchung teilnehmen.

$\mathrm{Zu}$ Beginn der Untersuchung müssen die Teilnehmer einen Stimmungsfragebogen beantworten und die akustischen und/oder raumklimatischen Bedingungen bewerten. Anschließend folgte der n-back Test in Stufe $n=4$ und $n=5$. Nach dem n-back Test ist wiederum der Stimmungsfragebogen und die Bewertung der akustischen und raumklimatischen Bedingungen zu bearbeiten. Ergänzend dazu erfolgt die subjektive Beurteilung des n-back Tests durch die Teilnehmer. Danach folgt das Ultimatumspiel. Die Reihenfolge der beiden Teile des Ultimatumspiels ist über die Teilnehmer hinweg ausbalanciert. Abschließend sind nochmals der Stimmungsfragebogen und die Bewertung der akustischen und raumklimatischen Bedingungen auszufüllen. Zum Abschluss erfolgt mit Hilfe einer vorgetäuschten Zusatzaufgabe die Untersuchung der Hilfsbereitschaft der Teilnehmer. Dafür werden die Teilnehmer in einem standardisierten Text, der den Teilnehmern am PC präsentiert wird, gefragt, ob sie noch freiwillig am Pauli-Test teilnehmen möchten. In der Voruntersuchung erfolgte die Frage nach freiwilliger Teilnahme mündlich durch den Versuchsleiter.

Hatte ein Teilnehmer kein Interesse teilzunehmen, musste er bis zum Abschluss der Untersuchung an seinem Arbeitsplatz bleiben, durfte jedoch in dieser Zeit ins Internet gehen. Die ganze Untersuchung dauerte ca. 3,5 Stunden.

\subsection{Experimentelles Setting}

Die Untersuchung wurde im High Performance Indoor Environment Labor (HiPIE-Labor) des Fraunhofer Institut für Bauphysik in Stuttgart durchgeführt. ${ }^{1}$ Das

1 Wir danken dem Fraunhofer Institut für Bauphysik (IBP), insbesondere Herrn Prof. Leistner und Herrn Dr. Liebl, für die kostenfreie Überlassung der Experimentierräume und die freundliche Unterstützung. 
Labor besitzt eine quadratische Grundfläche mit einer Seitenlänge von 6,6 Metern sowie eine Höhe von 2,8 Metern.

Die Schallereignisse werden mit Hilfe der Software Nuendo der Version 5.5.0 Bulid 3.4.5 (32 Bit) in einem externen Kontrollraum abgespielt. Das Schallfeld im HiPIE-Labor wird über eine elektroakustische Anlage mit Hilfe des Programms Iosono Unit 2.4.4 (Controll Revision 11143, 2012-03-28) erzeugt, die auf dem Prinzip der Wellenfeldsynthese beruht. ${ }^{2}$ Insgesamt sind im HiPIE-Labor 34 Lautsprecherpanele an den Raumbegrenzungsflächen angeordnet. Um die Verkehrslärmsituation realistisch wirken zulassen, werden in den Untersuchungen ausschließlich die neun Lautsprecherpanele der Fensterseite des Raumes verwendet.

Die Beeinflussung der Raumtemperatur erfolgt über eine externe Anlage. Über die Lüftungsschächte kann speziell temperierte Luft aus einer externen Anlage in das HiPIE-Labor gelangen und somit die Raumtemperatur und -feuchte konstant gehalten werden.

Pro Testung nehmen vier Probanden (teils fünf Probanden) gleichzeitig teil. Die Kalibrierung des Schalles erfolgt in der Mitte des Raumes mit Hilfe eines Schallpegelmessgerätes vom Typ 2233 der Firma Bruel und Kjaer.

Die Bekleidung der Probanden ist standardisiert, und zwar so dass die durch die Bekleidung hervorgerufene Dämmung der Körperwärme ca. $0.83 \mathrm{CLO}^{3}$ (ASHRAE, 2004) beträgt.

\subsection{Design und Stichprobe}

Es wird ein zweifaktorielles Versuchsdesign durchgeführt. Der Faktor Hintergrundschall (between-subject Faktor) ist zweifach gestuft (ca. $70 \mathrm{~dB}(\mathrm{~A})$ ) und ca. 37dB(A)), was in etwa dem Schallpegel von Straßenverkehrslärm bei geöffnetem und geschlossenem Fenster entspricht. Der Faktor Temperatur (between-subject Faktor) ist ebenfalls zweifach gestuft $\left(22^{\circ} \mathrm{C}, 29^{\circ} \mathrm{C}\right)$. Gemäß diesem $2 \times 2$-Design liegen bei der Hauptuntersuchung vier unterschiedliche Untersuchungsbedingungen vor.

2 Wellenfeldsynthese ist ein räumliches Audiowiedergabeverfahren mit dem Ziel, virtuelle akustische Umgebungen zu schaffen. Die Synthese erzeugt Wellenfronten, die von einem virtuellen Punkt ausgehen. Dessen akustische Lokalisation ist nicht von der Zuhörerposition und auch nicht, wie bei den konventionellen Mehrkanalverfahren, von psychoakustischen Effekten wie der Phantomschallquellenbildung abhängig.

3 Die Einheit „clo“ (clothing) misst den Isolationswert (thermischen Widerstand) von Bekleidung. Bekleidung mit dem Isolationswert 1 clo lässt eine Wärmemenge von $23 \mathrm{~kJ} / \mathrm{h}$ pro $\mathrm{m}^{2}$ bei $1^{\circ} \mathrm{C}$ Temperaturdifferenz zwischen Innen- und Außenfläche der Bekleidung durch. 
Die Tabelle veranschaulicht den Versuchsplan dieser Untersuchung. In jeder der vier Untersuchungsbedingungen werden 20 Personen untersucht, die nach Zufall den Bedingungen zugeteilt wurden. An jedem Setting nehmen insgesamt vier bis fünf Personen gleichzeitig Teil.

Insgesamt nahmen 80 Personen - davon 51 Männer und 29 Frauen - an der Untersuchung teil. Hauptsächlich sind die Probanden Studierende der Universität Stuttgart. Der Altersmedian der Gesamtstichprobe beträgt Md=22 Jahre (Range: 18 - 40 Jahre).

Tab. Versuchsaufbau

\begin{tabular}{ccc}
\hline & \multicolumn{2}{c}{ Temperatur } \\
\cline { 3 - 3 } & $\begin{array}{c}22^{\circ} \mathrm{C} \\
\left(\bumpeq \mathrm{MW}=22,57^{\circ} \mathrm{C}\right)\end{array}$ & $\begin{array}{c}29^{\circ} \mathrm{C} \\
\left(\hat{=} \mathrm{MW}=29,04^{\circ} \mathrm{C}\right)\end{array}$ \\
\hline $\begin{array}{c}37 \mathrm{~dB}(\mathrm{~A}) \\
\mathrm{n}_{1}\end{array}$ & $\mathrm{n}_{3}$ \\
\hline $\begin{array}{c}(\hat{=} \text { Straßenverkehrslärm bei } \\
\text { geschlossenem Fenster })\end{array}$ & & \\
\hline $\begin{array}{c}\text { 70dB(A) } \\
\text { (气 Straßenverkehrslärm bei } \\
\text { geöffnetem Fenster })\end{array}$ & $\mathrm{n}_{2}$ & $\mathrm{n}_{4}$ \\
\hline
\end{tabular}

\section{$4 \quad$ Ergebnisse}

Bezüglich der Fehlerraten im n-back Test zeigt sich, dass die Faktoren Temperatur und Hintergrundschall teilweise in ihrer Wirkung interagieren. Bei Straßenverkehrsgeräuschen mit einem Schallpegel von $37 \mathrm{~dB}(\mathrm{~A})$ zeigt sich für beide $\mathrm{n}$-Stufen ein tendenziell leistungsmindernder Einfluss von Hitze $(\mathrm{p}<.07)$. Bei Straßenverkehrslärm mit 70dB(A) kann dagegen kein Effekt der Raumtemperatur nachgewiesen werden. Generell werden in Stufe $n=5$ hochsignifikant mehr Fehler gemacht als in Stufe $\mathrm{n}=4(\mathrm{p}<.01)$. Die Variation des Hintergrundschalls bewirkt dagegen keine generelle Veränderung der Fehlerraten (s. Abbildung 1). 


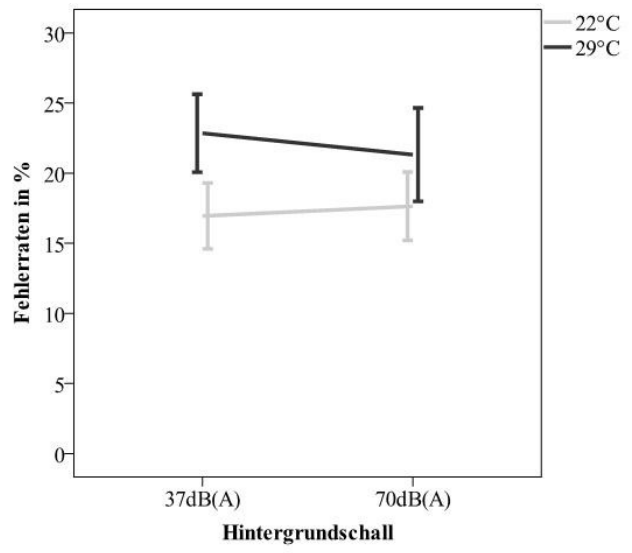

Abb. 1 Durchschnittliche Fehlerraten

Durchschnittliche Fehlerraten in Abhängigkeit der Faktoren Hintergrundschall, Temperatur in Stufe $n=5$. Dargestellt sind die Mittelwerte (MW) und die Standardfehler (SF) $\left(\mathrm{n}_{1}=\mathrm{n}_{2}=\mathrm{n}_{3}=\mathrm{n}_{4}=20\right)$.

Die Faktoren Temperatur und Hintergrundschall interagieren in ihrer Wirkung auf die Bearbeitungszeiten im n-back Test miteinander. Bei Straßenverkehrsgeräuschen mit $37 \mathrm{~dB}(\mathrm{~A})$ bewirkt die erhöhte Raumtemperatur in beiden $\mathrm{n}$-Stufen eine hochsignifikante Erhöhung der Bearbeitungszeiten (je p<.01) (s. Abbildung 2). Im Gegensatz dazu bewirkt die erhöhte Raumtemperatur bei Straßenverkehrsgeräuschen mit erhöhtem Schallpegel in Stufe $n=4$ keinen Effekt bzw. in Stufe $n=5$ ein Sinken der Bearbeitungszeiten ( $\mathrm{p}=.057$ ) (s. Abbildung 2). Insgesamt arbeiten die Probanden in Stufe $n=5$ hochsignifikant langsamer als in Stufe $n=4(p<.01)$. 


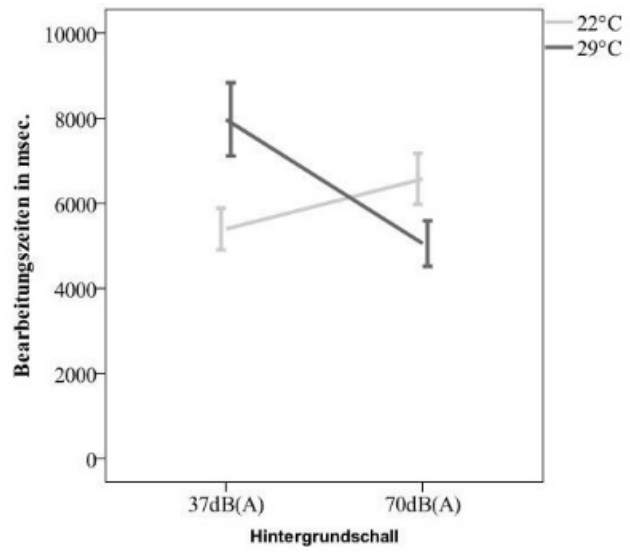

Abb. 2 Durchschnittliche Bearbeitungszeiten

Durchschnittliche Bearbeitungszeiten in Abhängigkeit der Faktoren Hintergrundschall, Temperatur in Stufe $n=5$. Dargestellt sind die Mittelwerte (MW) und die Standardfehler (SF) $\left(\mathrm{n}_{1}=\mathrm{n}_{2}=\mathrm{n}_{3}=\mathrm{n}_{4}=20\right)$.

Es zeigt sich im ersten Teil des Ultimatumspiels, dass die Fairness der Angebote direkte Auswirkung auf das Annahmeverhalten der Teilnehmer hat. Unabhängig von der jeweiligen Temperaturbedingung werden faire Angebote signifikant häufiger angenommen als unfaire Angebote $(\mathrm{p}<.05)$. Es kann jedoch weder ein Temperatur- noch ein Hintergrundschalleffekt oder interaktionistische Wirkungen auf das Annahmeverhalten der Probanden nachgewiesen werden.

Im zweiten Teil des Ultimatumspiels kann tendenziell ein Temperatureffekt aufgezeigt werden, da bei $70 \mathrm{~dB}(\mathrm{~A})$ Hintergrundschall die Angebote der Probanden der $22^{\circ} \mathrm{C}$-Bedingung tendenziell höher sind als die Angebote der Probanden der $29^{\circ} \mathrm{C}$-Bedingung $(\mathrm{p}=.083)$. Hinsichtlich des Faktors Hintergrundschall kann kein signifikanter Haupteffekt nachgewiesen werden.

Bei der freiwilligen Aufgabe am Schluss der Untersuchung (Pauli-Test) nehmen insgesamt hochsignifikant weniger Teilnehmer, die bei $29^{\circ} \mathrm{C}$ Temperatur getestet werden, teil als Probanden der $22^{\circ} \mathrm{C}$-Bedingung $(\mathrm{p}<.01)$. Des Weiteren zeigt sich, dass sowohl bei $37 \mathrm{~dB}(\mathrm{~A})$ als auch bei $70 \mathrm{~dB}(\mathrm{~A})$ Hintergrundschall mehr Teilnehmer der $22^{\circ} \mathrm{C}$-Bedingung freiwillig den Pauli-Test ausführen als Teilnehmer der $29^{\circ} \mathrm{C}$-Bedingung (je p<.05). Somit wirkt der Temperatureffekt unabhängig von dem 
jeweiligen Hintergrundschall. Hinsichtlich des Faktors Hintergrundschall kann kein Effekt auf die Teilnahmebereitschaft am Pauli-Test nachgewiesen werden.

Bei der Auswertung der Erregung zeigt sich zum Messzeitpunkt nach n-back eine fast signifikante Interaktion Hintergrundschall ${ }^{\star}$ Temperatur $(p=.057)$. Hier reduziert die erhöhte Raumtemperatur nur dann die Erregung hochsignifikant $(p<.01)$, wenn Straßenverkehrsgeräusche mit niedrigem Schallpegel im Hintergrund sind. Bei Straßenverkehrslärm bleibt die Temperaturwirkung zum Teil aus. Es kann keine generelle Wirkung des Faktors Hintergrundschall nachgewiesen werden. Jedoch sinkt bei $29^{\circ} \mathrm{C}$ Raumtemperatur die Erregung bei den Probanden, die unter $37 \mathrm{~dB}(\mathrm{~A})$ Hintergrundschall getestet wurden, signifikant stärker ab als bei den Probanden, die dem Straßenverkehrslärm von $70 \mathrm{~dB}(\mathrm{~A})$ ausgesetzt waren ( $<<.05)$ (s. Abbildung 3).

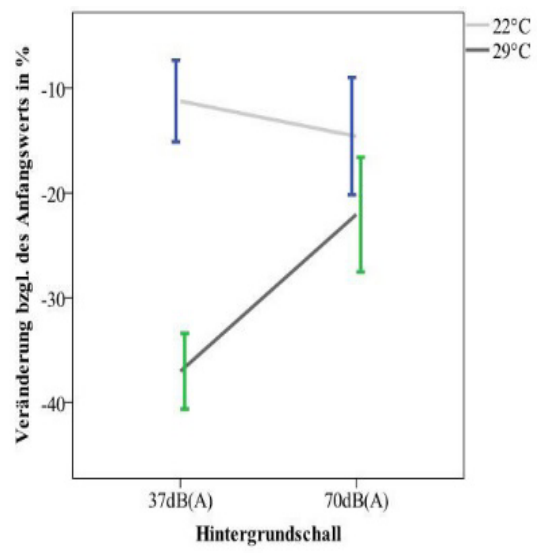

Abb. 3 Prozentuale Änderungswerte in der Skala Erregung

Prozentuale Änderungswerte bezüglich des Anfangswertes in der Skala Erregung zum Messzeitpunkt nach n-back in Abhängigkeit der Faktoren Temperatur und Hintergrundschall. Negative Änderungswerte kennzeichnen eine prozentuale Minderung der Erregung, positive Änderungswerte beschreiben eine prozentuale Steigerung. Dargestellt sind die Mittelwerte (MW) und die Standardfehler (SF) $\left(\mathrm{n}_{1}=\mathrm{n}_{2}=\mathrm{n}_{3}=\mathrm{n}_{4}=20\right)$.

Hinsichtlich der Verärgerung der Teilnehmer zeigt sich zum Messzeitpunkt nach n-back ansatzweise eine Interaktion der Faktoren Temperatur und Hintergrundschall. Bei Hintergrundschall von $37 \mathrm{~dB}(\mathrm{~A})$ zeigen die Probanden, die bei $29^{\circ} \mathrm{C}$ 
getestet werden, eine bedeutend stärkere Verärgerung als die Probanden, die bei $22^{\circ} \mathrm{C}$ getestet werden $(\mathrm{p}<.01)$. Bei $70 \mathrm{~dB}(\mathrm{~A})$ Hintergrundschall kann dagegen keine Wirkung des Faktors Temperatur nachgewiesen werden. Zum Messzeitpunkt Schluss kann dagegen nachgewiesen werden, dass Hitze die Verärgerung unabhängig des jeweiligen Hintergrundschalls erhöht. Des Weiteren führt Straßenverkehrslärm zum Messzeitpunkt nach n-back zu einer Minderung der Verärgerung, wenn die Raumtemperatur $29^{\circ} \mathrm{C}$ beträgt ( $<<.05$ ). Es zeigt sich, dass $29^{\circ} \mathrm{C}$ Raumtemperatur als signifikant unbehaglicher empfunden werden als $22^{\circ} \mathrm{C}(\mathrm{p}<.01)$, unabhängig vom jeweiligen Hintergrundschall.

$\mathrm{Zu}$ den Messzeitpunkten Anfang und nach n-back mindert die Hitze den Raumkomfort und die Zufriedenheit der Teilnehmer, zum Messzeitpunkt Schluss kann jedoch kein Temperatureffekt nachgewiesen werden. Der Faktor Temperatur wirkt zum Messzeitpunkt nach n-back belästigend ( $\mathrm{p}<.01)$ auf die Probanden, zum Messzeitpunkt Schluss jedoch nicht. Dies erklärt die hochsignifikante Interaktion Messzeitpunkt ${ }^{\star}$ Temperatur $(\mathrm{p}<.01)$. Der Faktor Hintergrundschall wirkt sich insofern aus, als dass $29^{\circ} \mathrm{C}$ Raumtemperatur bei Hintergrundschall mit niedrigem Schallpegel tendenziell $(\mathrm{p}=.087)$ lästiger empfundenen werden als bei Hintergrundschall mit hohem Schallpegel.

Straßenverkehrslärm mit hohem Schallpegel wird generell als lästiger empfunden als Straßenverkehrsgeräusche mit niedrigem Schallpegel $(\mathrm{p}<.01)$. Wider Erwarten kann ein tendenzieller Temperatureffekt nachgewiesen werden, der sich darin äußert, dass Straßenverkehrsgeräusche mit niedrigem Schallpegel bei $29^{\circ} \mathrm{C}$ ansatzweise als lästiger empfunden werden als bei $22^{\circ} \mathrm{C}(\mathrm{p}=.087)$.

Es kann nachgewiesen werden, dass Straßenverkehrsgeräusche mit erhöhtem Schallpegel die Zufriedenheit der Probanden unabhängig von der jeweiligen Raumtemperatur verschlechtert $(\mathrm{p}<.01)$. Desweiteren zeigt sich, dass Straßenverkehrslärm mit $70 \mathrm{~dB}(\mathrm{~A})$ als konzentrationsstörender empfunden werden als Straßenverkehrsgeräusche mit $37 \mathrm{~dB}(\mathrm{~A})(\mathrm{p}<.01)$ (s. Abbildung 4). 


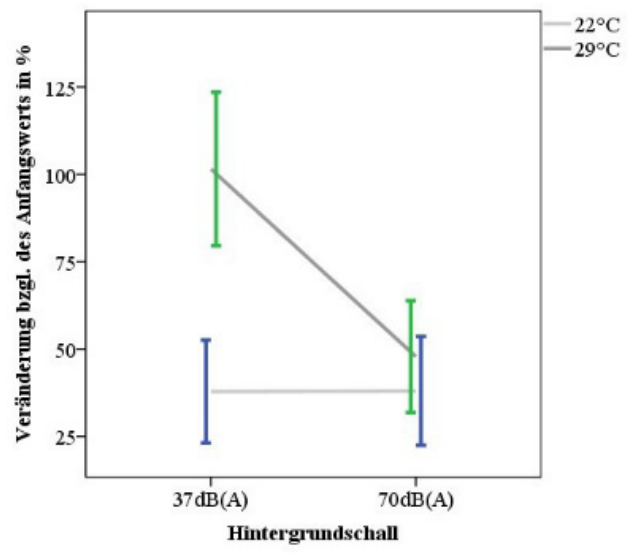

Abb. 4 Prozentuale Änderungswerte in der Skala Verärgerung

Prozentuale Änderungswerte bezüglich des Anfangswertes in der Skala Verärgerung zum Messzeitpunkt nach n-back in Abhängigkeit der Faktoren Temperatur und Hintergrundschall. Dargestellt sind die Mittelwerte (MW) und die Standardfehler (SF) $\left(\mathrm{n}_{1}=\mathrm{n}_{2}=\mathrm{n}_{3}=\mathrm{n}_{4}=20\right)$.

\section{Diskussion}

Die Ergebnisse der laborexperimentellen Untersuchung zeigen, dass Hitze die Hilfsbereitschaft der Teilnehmer reduziert. Zudem werden Interaktionen zwischen den Faktoren Temperatur und Straßenverkehrslärm bezüglich des Einflusses auf kognitive Leistung, Bearbeitungszeiten, Stimmung sowie subjektiver Bewertung nachgewiesen.

Es hat sich gezeigt, dass die Faktoren Temperatur und Hintergrundschall in ihrer Wirkung auf Leistung interagieren. In ruhiger Umgebung führt Hitze tendenziell zur Leistungsverschlechterung. Dies ist konform zu den Ergebnissen von Häggblom, Hongisto, Haapakangas \& Koskela (2011). In deren Studie waren die Leistungen der Probanden im n-back Test bei $29^{\circ} \mathrm{C}$ Raumtemperatur ebenfalls reduziert. In dieser Untersuchung kann dagegen bei Straßenverkehrslärm mit 70dB(A) kein Effekt der Raumtemperatur nachgewiesen werden. Man kann hier einen antagonistischen Effekt erkennen, bei dem die Einzelstressoren entgegengesetzt wirken und sich gegenseitig aufheben. 
Die Tatsache, dass dem Faktor Hintergrundschall generell keine Wirkung zugesprochen werden kann, zeigt, dass der Einfluss der Hitze dominiert. Dies kann an der unterschiedlichen Art und Weise liegen, wie die Stressoren verarbeitet werden. Temperatur wirkt primär physisch sowie - nach entsprechender Bewertung - emotional und kognitiv. Lärm dagegen beeinflusst primär das kognitive System des Menschen. Man kann sich bei Leistungstests demnach dem Lärm besser entziehen, zum Beispiel durch Konzentrationserhöhung. Bei Hitze gelingt dies nicht ohne weiteres, da sie physisch belastender wirkt als Lärm. Eventuell war die kognitive Belastung des hier verwendeten Straßenverkehrslärms nicht groß genug um letztendlich zur Leistungsminderung zu führen. In der subjektiven Bewertung dagegen zeigt sich, dass der Lärm als Störquelle wahrgenommen wird.

In ihrer Wirkung auf die Bearbeitungszeiten im n-back Test interagieren die Faktoren Temperatur und Hintergrundschall ebenfalls. In ruhiger Umgebung verlangsamen sich bei den Probanden, die unter erhöhter Raumtemperatur getestet werden, die Bearbeitungszeiten. Im Gegensatz dazu bewirkt Hitze bei Straßenverkehrslärm keinen Effekt bzw. schnellere Bearbeitungszeiten. Hitze und Straßenverkehrslärm wirken somit entgegengesetzt. Es kann angenommen werden, dass die Probanden, die unter Hitze und Straßenverkehrslärm getestet werden, so belastet sind, dass sie durch schnelleres Arbeiten der Situation entfliehen wollen.

Im Hinblick auf die Stimmungskomponente Erregung kommt es beim Messzeitpunkt nach n-back zu Interaktionen der beiden Faktoren, da Hitze nur dann die Erregung reduziert, wenn Straßenverkehrsgeräusche mit niedrigem Schallpegel im Hintergrund sind. Bei Straßenverkehrslärm verändert Hitze die Erregung nicht. Zudem erhöht der Straßenverkehrslärm die Erregung dann, wenn die Raumtemperatur hoch ist. Man sieht somit, dass entsprechend der Arousaltheorie (Hygge, 1991) Straßenverkehrslärm Erregung steigert und Hitze Erregung reduziert. In Zusammenwirken der Stressoren kommt es folglich zu einem mittleren Leistungsniveau. Dies ist im Sinne der umgekehrten U-Funktion leistungsoptimierend, was die antagonistischen Effekte beim n-back Test bestätigt.

Bei den Ergebnissen der Skala Verärgerung kann ebenfalls eine antagonistische Interaktion zwischen den beiden Stressoren nachgewiesen werden. Bei 37dB(A) Schallpegel führt Hitze zu einer Erhöhung der Werte in der Skala Verärgerung. Im Gegensatz dazu reduziert Straßenverkehrslärm in Kombination mit Hitze die Verärgerung. Zur Begründung kann das „Negative Affect-Escape Model“ (Baron \& Bell, 1976) herangezogen werden. Demzufolge löst Hitze negative Emotionen in Form von Verärgerung aus. Man vermutet einen kurvilinearen Zusammenhang zwischen Hitze und negativen Emotionen im Sinne einer umgekehrten-U-Funktion. Hitze lässt die Verärgerung bis zu einem kritischen Punkt ansteigen. Kommt nun zusätzlich Straßenverkehrslärm hinzu, so wird der kritische Punkt der Belastung 
überschritten, so dass schließlich die Verärgerung wieder abnimmt. In kombinierter Belastung durch Lärm und Hitze scheinen die Personen mehr darauf konzentriert zu sein, der Situation zu entkommen als Verärgerung zu zeigen.

Es hat sich gezeigt, dass Hitze die Bereitschaft zu freiwilligem Hilfsverhalten in der Untersuchung reduziert. Der Straßenverkehrslärm bewirkt keinen Effekt. Die Dominanz der Hitze gegenüber dem Straßenverkehrslärm kann ebenfalls durch die unterschiedlichen Verarbeitungsmechanismen der beiden Stressoren erklärt werden. Hitze belastet auf körperlicher, emotionaler und kognitiver Ebene und ist somit in ihrem Einfluss umfassender als Straßenverkehrslärm, welcher das kognitive System belastet. Es ist somit zu vermuten, dass die Probanden der Hitze im Labor entkommen wollten und deshalb weniger freiwillig Hilfsbereitschaft gezeigt haben als die Probanden bei $22^{\circ} \mathrm{C}$.

\section{$6 \quad$ Zusammenfassung und Ausblick}

Studien und Prognosen weisen auf einen Anstieg der Straßenverkehrslärmbelastung und vermehrte Hitzeperioden in den kommenden Jahrzehnten hin. Die Ergebnisse dieser Untersuchung liefern Hinweise darauf, dass Hitze in Kombination mit Straßenverkehrslärm die Arbeitsleistung qualitativ und quantitativ verschlechtern, emotional belasten und zu Konflikten im sozialen Miteinander führen kann. Diese Erkenntnisse bieten Entscheidungshilfen beim Ergreifen von Lärm- und Hitzestress vorbeugenden Maßnahmen. Schutzmaßnahmen gegen Lärm und Hitze sollten neben der Optimierung der Arbeitsleistung auch emotionale und soziale Wirkungsweisen der Stressoren berücksichtigen.

\section{Literatur}

Anderson, C.A., Anderson, K.B., Dorr, N., DeNeve, K.M. \& Flanagan, M. (2000). Temperature and aggression. Advances in Experimental Social Psychology, 32: 63-133.

Anderson, C.A., Deuser, W.E. \& DeNeve, K. (1995). Hot temperatures, hostile affect, hostile cognition, and arousal: Tests of a general model of affective aggression. Personality and Social Psychology Bulletin, 21: 434-448.

Arnold, W. (1975). Der Pauli Test: Anweisung zur sachgemäßen Durchführung, Auswertung und Anwendung des Kraepelinschen Arbeitsversuches. Berlin: Springer. 
ASHRAE (2004). ANSI/ASHRAE standard 55-2004, Thermal environmental conditions for human occupancy. Atlanta: American Society of Heating, Refrigerating and Airconditioning Engineers; 2004. 27 p.

Babisch, W. (2006). Transportation Noise and Cardiovascular Risk Review and Synthesis of Epidemiological Studies Dose-effect Curve and Risk Estimation (WaBoLu-Hefte 01/06). Berlin: Umweltbundesamt.

Baron, R.A. \& Bell, P.A. (1975). Aggression and Heat: Mediating Effects of Prior Provocation and Exposure to an Aggressive Model. Journal of Personality and Social Psychology, 31(5): 825-832.

Baron, R.A. \& Bell, P.A. (1976). Aggression and Heat: The Influence of Ambient Temperature, Negative Affect, and a Cooling Drink on Physical Aggression. Journal of Personality and Social Psychology, 33(3): 245-255.

Bell, C.R., Provins, K.A. \& Hiorns, R.W. (1964). Visual and Auditory Vigilance During Exposure to Hot and Humid Conditions. Ergonomics, 7: 279-288.

Bell, P.A. \& Doyle, D.P. (1983). Effects of heat and noise on helping. Psychological Reports, 53: 955-959.

Bell, P.A. (1980). Effects of Heat, Noise, and Provocation on retaliatory evaluative Behavior. Journal of Social Psychology, 110: 97-100.

Bluhm G, Nordling E, Berglind N. (2001). Increased prevalence of hypertension in apopulation exposed to road traffic noise. In: Internoise 2001 Proceedings of the 2001 international congress and exhibition on noise control engineering: R. Boone (Hrsg.), (pp. 1563-1566). Maastricht: Nederlands Akoestisch Genootschap.

Broadbent, D.E. (1971). Decision and Stress. New York: Academic Press.

Carlsmith, J.M. \& Anderson, C.A. (1979). Ambient temperature and the occurrence of collective violence: A new analysis. Journal of Personality and Social Psychology, 37: 337-344.

Clausen, G., Carrick, L., Fanger, P.O., Kim, S.W., Poulsen, T. \& Rindel, J.H. (1993). A Comperative Study of Discomfort caused by Indoor Air Pollution, Thermal Load and Noise. Indoor Air, 3: 255-262.

Cohen, S. \& Spacapan, S. (1984). The social psychology of noise. In: D.M. Jones \& A.J. Chapman (Hrsg.), Noise and society (pp. 221-245). Chichester: Wiley.

Coumou, D. \& Robinson, A. (2013). Historic and future increase in the global land area affected by monthly heat extremes. Environmental Research Letters, 8: 034018.

Cunningham, M.R. (1979). Weather, mood, and helping behavior. Quasi experiments with the sunshine Samaritian. Journal of Personality and Social Psychology, 37: 1947-1956.

Donnerstein, E. \& Wilson, D.W. (1976). Effects of noise and perceived control on ongoing and subsequent aggressive behavior. Journal of Personality and Social Psychology, 34: 774-781.

Fahrenberg, J., Hampel, R. \& Selg, H. (2001). Das Freiburger Persönlichkeitsinventar FPI-R. 7. Aufl. Göttingen: Hogrefe.

Fields, J.M. (1993). Effect of personal and situational variables on noise annoyance in residential areas. Journal of the Acoustical Society of America, 93(5): 2753-2763.

Geen, R.G. \& O’Neal, E.C. (1969). Acitivation of cue-elicted aggression by general arousal. Journal of Personality and Social Psychology, 11: 289-292.

Grether, W.F. (1970). Effects on human performance of combined environmental stresses. Aerospace Medicine Technical Report, 68: 70-71.

Griefahn, B. (2000). Noise-induced extra aural effects. Journal of the Acoustical Society Japan, 21(E): 307-317. 
Gunnarsen, L.B., \& Santos, A.M.B. (1998). Relative importance of temperature, draft, noise and window area in offices. I Proceedings of the EPIC' 98, the 2nd European Conference on Energy Performance and Indoor Climate in Buildings. (Vol. 3, pp. 862-867).

Guski, R. (1999). Personal and social variables as co-determinants of noise annoyance. Noise und Health, 3: 45-65.

Häggblom, H., Haapakangas, A., Hongisto, V. \& Koskela, H. (2011). The effect of temperature on work performance and thermal comfort - laboratory experiment. In: The 12th International Conference on Indoor Air Quality and Climate (paper 226). Austin, TX.

Hancock, P.A. \& Pierce, J.O. (1985). Combined Effects of Heat and Noise on Human Performance: A Review. American Industrial Hygiene Association Journal, 46(10): 555-566.

Hancock, P.A., Ross, J.M. \& Szalma, J.L. (2007). A Meta-Analysis of Performance Response Under Thermal Stressors. Human Factors, 49(5): 851-877.

Hellbrück, J. \& Liebl, A. (2008). Noise effects on cognitive performance. In: S. Kuwano (Hrsg.), Recent topics in environmental psychoacoustics (pp. 153-184). Osaka: Osaka University Press.

Hellbrück, J., Guski, R. \& Schick, A. (2010). Schall und Lärm. In: V. Linneweber, E.D. Lantermann \& E. Kals (Hrsg.), Umweltpsychologie (Bd. 2, Spezifische Umwelten und umweltbezogenes Handeln. Enzyklopädie der Psychologie) (hrsg. von N. Bierbaumer et al.) (pp. 3-44). Göttingen: Hogrefe.

Horie, G., Sakurai, Y., Nogushi, T. \& Matsubara, N. (1985). Synthesized evaluation of noise, lighting and thermal conditions in a room. In: Proceedings of Noise Control 85, International Conference on Noise Control Engineering (pp. 491-496). Krakau.

Hübler, M. \& Klepper, G. (2007). Kosten des Klimawandels: Die Wirkung steigender Temperaturen auf Gesundheit und Leistungsfähigkeit. Hamburg: WWF Deutschland Frankfurt.

human occupancy. Atlanta: American Society of Heating, Refrigerating and Airconditioning.

Hygge, S. \& Knez, I. (2001). Effects of noise, heat and indoor lighting on cognitive performance and self-reported affect. Journal of Enviromental Psychology, 21(3): 291-299.

Hygge, S. (1991). The interaction of noise and mild heat on cognitive performance and serial reaction time. Environment International, 17: 229-234.

Hygge, S., Boman, E. \& Enmarker, I. (2003). The effects of roadtraffic noise and meaningful irrelevant speech on different memory systems. Scandinavian Journal of Psychology 44: 13-21.

Intraplan Consult GmbH und BVU Beratergruppe Verkehr + Umwelt GmbH (2007). Prognose der deutschlandweiten Verkehrsverflechtungen 2025, im Auftrag des Bundesministeriums für Verkehr, Bau und Stadtentwicklung (FE-Nr. 96.0857/2005). München/Freiburg.

Janke, W. \& Debus, G. (1978). Die Eigenschaftswörterliste: EWL; eine mehrdimensionale Methode zur Beschreibung von Aspekten des Befindens. Göttingen: Hogrefe.

Job, R.F.S. (1988). Community response to noise: A review of factors influencing the relationship between noise exposure and reaction. Journal of the Acoustical Society of America, 83(3): 991-1001.

Koneci, V.J., Libuser, L., Morten, H. \& Ebbsen, E.B. (1975). Effects of violation of personal space on escape and helping responses. Journal of Personality and Social Psychology, 11: 288-299.

Korte, C. \& Grant, R. (1980). Traffic noise, environmental awareness, and pedestrian behavior. Environment and Behaviour, 12: 408-420.

Krahé, B. (2007). Aggression. In: K. Jonas, M. Hewstone \& W. Stroebe (Hrsg.), Sozialpsychologie (pp. 265-294). Heildelberg: Springer. 
Mathews, K.E. \& Canon, L.K. (1975). Environmental noise level as a determinant of helping behavior. Journal of Personality and Social Psychology, 32: 571-577.

Murray, R.H. \& McCally, M.M. (1973). Combined environmental stresses. In: J.F. Parker Jr. \& V.R. West (Hrsg.), Bioastronautics Data Book. 2. Aufl. NASA SP-3006 (pp. 881-914). Washington, DC: US Government Printing Office.

Page, R.A. (1978). Environmental influences on prosocial behavior: The effect of temperature. Paper presented to the Midwestern Psychological Association. Chicago.

Pellerin, N. \& Candas, V. (2003). Combined effects of temperature and noise on human discomfort. Physiological Behavior 78: 99-106.

Pellerin, N. \& Candas, V. (2004). Effects of steady-state noise and temperature conditions on environmental perception and acceptability. Indoor Air, 14: 129-136.

Pepler, R.D. (1960). Warmth, Glare and a background of Quiet Speech: A Comparision of Their Effects on Performance. Ergonomics, 3: 68-73.

Pilcher, J.J., Nadler, E. \& Busch, C. (2002). Effects of hot and cold temperature exposure on performance: a meta-analytic review. Ergonomics, 45(10): 682-698.

Postle, B.R., D'Esposito, M. \& Corkin, S. (2005). Effects of verbal and nonverbal interference on spatial and object visual working memory. Memory und Cognition, 33(2): 203-212.

Renshaw, F. (1971). Combined Effects of Heat and Noise on Work Perfomance. Am. Ind. Hyg. Assoc.J., Abstr. Suppl. 3: 38.

Rückert-John, J., Bormann, I. \& John, R. (2013). Umweltbewusstsein in Deutschland 2012: Ergebnisse einer repräsentativen Bevölkerungsumfrage. Berlin/Marburg: Bundesministerium für Umwelt, Naturschutz und Reaktorsicherheit und Umweltbundesamt und Umweltbundesamt.

Schneider, F.W., Lesko, W.A. \& Gerrett, W.A. (1980). Helping behavior in hot, comfortable, and cold temperatures. Enviroment und Behavior, 12: 231-240.

Schütte, M. \& Marks, A. (2004). Entwicklung des Dortmunder Lärmempfindlichkeits-Fragebogens (DoLe). Paper präsentiert bei Arbeit + Gesundheit in effizienten Arbeitssystemen. Dortmund.

Schwenkmezger, P., Hodapp, V. \& Spielberger, C.D. (1992). Das State-Trait-Ärgerausdrucks-Inventar STAXI. Göttingen: Hogrefe.

Seppänen, O., Fisk, W.J., \& Faulkner, D. (2004). Control of temperature for health and productivity in offices (No. LBNL-55448). Berkeley, CA: Lawrence Berkeley National Laboratory.

Seppänen, O., Fisk, W.J. \& Lei, O.H. (2006). Effect of Temperature on Task Performance in Office. (No. LBNL-60946). Berkeley, CA: Lawrence Berkeley National Laboratory.

Sherrod, D.R. \& Downs, R. (1974). Environmental determinants of altruism: The effects of stimulus overload and perceived control on helping. Journal of Personality and Social Psychology, 10: 468-479.

Tinz, B., Freydank, E. \& Hupfer, P. (2008). Hitzeepisoden in Deutschland im 20. und 21. Jahrhundert. In: H. Graßl, G. Jendritzky, L. Karbe, J.L. Lozán \& K. Reise (Hrsg.), Warnsignale. Warnsignal Klima: Gesundheitsrisiken. Gefahren für Menschen, Tiere und Pflanzen; wissenschaftliche Fakten; mit 37 Tabellen (pp. 141-148). Hamburg: Wiss. Auswertungen.

Umweltbundesamt (UBA) (2010). Zusammenstellung der Mitteilungen der Bundesländer sowie des Eisenbahn-Bundesamtes entsprechend $\$ 47 \mathrm{c}$ BImSchG.

Viteles, M. \& Smith, K.R. (1946). An experimental investigation of the eject of change in atmospheric conditions and noise upon performance. Heating, Piping und Air Conditioning, 18: 107-112. 
Werner, J. (2005). Regulatory processes of the human body during thermal and work strain. In: Y. Tochihara \& T. Ohnaka (Hrsg.), Elsevier ergonomics book series. Environmental ergonomics. The ergonomics of human comfort, health, and performance in the thermal environment (pp. 3-15). Amsterdam/Oxford: Elsevier.

Witterseh, T., Wyon, D.P. \& Clausen, G. (2004). The effects of moderate heat stress and open-plan office noise distraction on SBS symptoms and on the performance of office work. Indoor Air, 14(8): 30-40.

Zebisch, M., Grothmann, T., Schröder, D., Hasse, C., Fritsch, U. \& Cramer, W. (2005). Klimawandel in Deutschland - Vulnerabilität und Anpassungsstrategien klimasensitiver Systeme. Potsdam-Institut für Klimafolgenforschung im Auftrag des Umweltbundesamts. Climate Change, 8: 122-136.

Open Access Dieses Kapitel wird unter der Creative Commons Namensnennung - Nicht kommerziell 2.5 International Lizenz (http://creativecommons.org/licenses/by-nc/2.5/deed. de) veröffentlicht, welche für nicht kommerzielle Zwecke die Nutzung, Verbreitung und Wiedergabe in jeglichem Medium und Format erlaubt, sofern Sie den/die ursprünglichen Autor(en), den Titel des Werks und die Quelle ordnungsgemäß nennen, einen Link zur Creative Commons Lizenz beifügen und im Falle einer Abwandlung durch einen entsprechenden Hinweis deutlich erkennbar machen, dass Änderungen vorgenommen wurden.

Die in diesem Kapitel enthaltenen Bilder und sonstiges Drittmaterial unterliegen ebenfalls der genannten Creative Commons Lizenz, sofern sich aus der Abbildungslegende nichts anderes ergibt. Sofern das betreffende Material nicht unter der genannten Creative Commons Lizenz steht und die betreffende Handlung nicht nach gesetzlichen Vorschriften erlaubt ist, ist auch für die oben aufgeführten nicht-kommerziellen Weiterverwendungen des Materials die Einwilligung des jeweiligen Rechteinhabers einzuholen. 